\title{
EL AEROPUERTO DE EL ALTET Y SUS PERSPECTIVAS DE FUTURO
}

\author{
Enrique Matarredona Coll ${ }^{1}$
}

\section{RESUMEN}

Los objetivos del presente trabajo son dos: en principio, se pretende analizar los parámetros que definen en la actualidad al tráfico aéreo y sus perspectivas de futuro, y a continuación, se realiza un diagnóstico sobre el pasado, presente y futuro del aeropuerto de El Altet en el que el autor propone una serie de reflexiones y consideraciones tendentes a dinamizar una infraestructura que debe ser considerada como pieza fundamental para el mantenimiento y posterior desarrollo del sector turístico alicantino.

Palabras clave: Tráfico aéreo - Aeropuerto - Análisis geográfico - Ordenación territorial - Planificación - Turismo.

\begin{abstract}
The objectives of this work are two: at first, it is tried to analyse the parameters which define nowadays the aerial traffic and its future perspectives, and then, it is achieved a diagnosis about past, present and future of «El Altet» airport where the author proposes some reflections and considerations which lead up to invigorate an infrastructure which we have to consider as a fundamental piece for the touristic sector from Alicante.

Key words: Aerial Traffic - Airport - Geographic Analysis - Territorial Management - Planning - Tourism.
\end{abstract}

El presente trabajo pretende realizar, por un lado, un breve análisis del transporte aéreo y lo que representa en el mundo actual; y por otro, una aproximación al conocimiento del aeropuerto de El Altet, su área de influencia, la evolución global del tráfico - tanto de pasajeros como de mercancías-, la relación del fenómeno charter con el turismo de la Costa Blanca, así como, las perspectivas de futuro del aeropuerto alicantino sobre el que se ciernen «negros nubarrones» y complejas soluciones que, en los últimos meses, han sido

1 El presente trabajo contiene parte del texto utilizado por el autor en la conferencia pronunciada el día 9 de diciembre de 1992 en ocasión del Congreso sobre «Presente y futuro del transporte aéreo en Alicante», organizado por el Excmo. Ayuntamiento de esta ciudad. Agradezco las sugerencias realizadas por el prof. MORALES GIL, que han permitido la redacción de alguna de las ideas aquí vertidas. 
objeto de reflexión y discusión por parte de los distintos estamentos administrativos, políticos y sociales, provinciales y locales.

\section{El transporte aéreo}

En lo que hace referencia al primer punto, es preciso indicar que en España, a tenor de lo que reflejan las estadísticas publicadas por el INE y los estudios monográficos relativos a este tema (CANO, 1980) ${ }^{2}$, se puede confirmar una transferencia, hacia el sistema aéreo, de tráficos y pasaje procedentes de otros medios de transporte; transferencia que puede relacionarse con el aumento del nivel económico y la estructura tarifaria, aunque su importancia a nivel global todavía sea bastante reducida.

La explicación del trasvase o transferencia aducida reside en ciertas ventajas que el transporte aéreo tiene sobre los demás. En este sentido, WOLKOWITSCH $(1973)^{3}$ alude a la evitación de cambios sucesivos de sistemas, DACHARRY (1981) menciona la facilidad de carga, LABASSE (1973) concreta la competencia en distancias y matiza el tipo de flete más adecuado, SAILE (1975) señala la velocidad de desplazamiento, y, consiguientemente, el tiempo como factor esencial, pese al punto negativo que puede suponer el problema de los accesos o las interferencias meteorológicas. La comodidad y mejores servicios, la publicidad y la moderna infraestructura son algunos de los «factores añadidos» aducidos por los defensores del sistema aéreo. BELOTTI (1975) $)^{4}$, aparte de remarcar el papel del transporte aéreo en servicios urgentes, sociales o científicos y la aportación en el movimiento turístico y comercio internacional, señala la flexibilidad en el uso de la flota que permite el intercambio entre distintos espacios y épocas en función de la demanda.

La ocupación y ordenación del espacio realizadas por el transporte aéreo no se lleva a cabo sin competencia y ésta a dos niveles: uno, entre los distintos medios de transporte, y otro, la concurrencia empresarial dentro del aéreo. LABASSE $(1973)^{5}$ y DACHARRY $(1981)^{6}$ previenen sobre los riesgos que entraña el establecer criterios comparativos en este sentido o determinar ventajas y desventajas de unos y otros. Con todo, según los criterios de los investigadores en el campo de la planificación, la competencia entre el transporte aéreo y otros medios puede repercutir en el rendimiento de un determinado aeropuerto, pero no por ello debe tener incidencia a nivel global.

En el análisis de la competencia con otros medios de comunicación se manejan frecuentemente umbrales de distancia. LABASSE (1973) destaca su ventaja en el transporte de viajeros a partir de $500 \mathrm{~km}$, y sobre todo desde los $1.000 \mathrm{~km}$; BELOTTI (1975) marca la supremacía del avión por encima de los $800 \mathrm{~km}$, con referencia, por ejemplo al tren, aunque hoy en día la competencia de los coches-cama y los trenes de alta velocidad sea importante. A pesar de todo, hemos de admitir que estos umbrales kilométricos varían según las distintas realidades geográficas.

En el estudio competetitivo, las distancias adquieren un verdadero valor traducidas en tiempo, y éste, a su vez, debe cuantificarse en precio, si bien el resultado final no es fácil de determinar de modo absoluto por la cantidad de variables a tener en cuenta. En los «tiempos» por vía aérea se ha de considerar no sólo el vuelo, sino también el trayecto de

2 CANO GARCÍA, G., (1980): El transporte aéreo en España, Ariel, Barcelona.

3 WOLKOWITSCH, M., (1973): Geographie des transports, Colin, París.

4 BELOTTI, J., (1975): L'economie du transport aérien, Belotti, Chatillon.

5 LABASSE, J., (1973): La organización del espacio, IEAL, Madrid.

6 DACHARRY, M., (1981): Geographie du transport aérien, Litec, París. 
superficie y las formalidades administrativas a cumplir en el embarque y desembarque. Y cada uno de estos componentes depende de una serie de circunstancias: velocidad de crucero de la aeronave, características del aeropuerto, formalidades según el tipo de servicio aéreo, trayecto de superficie, accesos al aeropuerto, etc.

Ahora bien, ni aún teniendo en cuenta los tiempos globales en relación con otro medio, se pueden establecer criterios de competencia, porque el valor real depende de toda una serie de circunstancias (tipo de viajeros, momento del viaje, finalidad, temor de mayores riesgos en el avión, accesos, enlaces con otros sistemas), que hacen difícil tal valoración. En definitiva, se trataría de la valoración precio o valoración tiempo. La comparación entre diferentes medios de transporte con diferentes velocidades y tarifas permite afirmar que el factor distancia no lo explica todo, pues la infraestructura ferroviaria y aérea existentes tienen mucho que ver en ello.

Aparte de las diferencias de desarrollo económico espacial, otras consideraciones geográficas modifican el criterio de la distancia, como pueden ser cuestiones topográficas, densidad de infraestructuras de los sistemas de competencia o motivaciones turísticas. Otro factor, que matiza distancia y tiempo, es el horario (según sean viajes diurnos o nocturnos) o ciertos servicios adicionales (posibilidad de embarque de automóviles en sistemas terrestres o marítimos). Todo ello, en definitiva, nos lleva a la conclusión de que la distancia no es el único factor de competencia ni debe manejarse con límites rígidos, sino que entran en juego el espacio geográfico y una serie de circunstancias adicionales distintas al simple kilometraje.

Por otro lado, conviene mencionar las repercusiones del fenómeno turístico en la competencia interior, tanto por la canalización que realizan la agencias como por los apretados programas de visitas que requieren un sistema rápido de transporte.

En definitiva, y a otro nivel, cabe esperar en el futuro un transporte aéreo distinto con aviones aptos para cortas distancias, de fácil despegue y aterrizaje, que economicen combustible y simplifiquen infraestructuras. Tales fórmulas podrían ser la respuesta a las necesidades del progreso y posibilitarían quizás unos costes más racionales.

\section{Características básicas del aeropuerto de El Altet}

Con el fuerte crecimiento del tráfico, los grandes aeropuertos ocupan, en el espacio geográfico sobre el que se ubican, una superficie que no cesa de incrementarse. En la actualidad, se estima que el índice o relación superficie/tráfico puede estar en 100 ha/un millón de pasajeros anual o 100.000 Tm de fletes mercantiles. Suelen situarse en la proximidad de la ciudad de la que depende, pero sus necesidades de espacio y sus índices de contaminación ambiental — sobre todo, ruidos - tienden a hacerlos instalarse lejos de los núcleos urbanos, con la consiguiente pérdida de competitividad emanada de tal circunstancia. Requieren accesos terrestres fáciles y rápidos, no sólo para el usuario del transporte sino también para los empleados del aeropuerto.

Los usuarios pueden agruparse en tres categorías: viajeros de negocios, turistas y expedidores de mercancías. Los que van de viaje comercial consideran fundamentalmente el tiempo como algo primordial, por lo que la rapidez de circulación entre las terminales aéreas y los centros de las ciudades se considera imprescindible. Los turistas son menos exigentes en cuanto al tiempo salvo que vengan en fin de semana, que es una de las modalidades de turismo que se quiere fomentar durante el invierno en la Costa Blanca (sobre todo en Benidorm). Los expedidores de mercancías perecederas demandan, asimismo, agilidad en la proximidad de las terminales de carga. 
Los segundos, los trabajadores del aeropuerto, necesitan fluidez en la circulación de los accesos, ya que constituyen un colectivo bastante importante —unos 1.100 por cada millón de pasajeros-, de forma que en horas punta contribuyen con su desplazamiento al trabajo a un incremento del tráfico, a pesar de que normalmente, parte de ellos, localizan sus viviendas en las cercanías de estas vías y del aeropuerto.

De todo ello, se deduce que el planteamiento teórico de la ordenación de los accesos a los aeropuertos exige fluidez y rapidez, porque de otra forma cualquier instalación de este tipo está condenada a muerte por inanición.

La autovía ha sido considerada como el medio de acceso más flexible y económico, al tiempo que más conforme a la rapidez deseada por los usuarios. Se presta tanto al transporte individual como colectivo. Por otro lado, a la hora de establecer posibles alternativas, el sistema férreo es a menudo criticado por sus frecuentes insuficiencias, estimándose que el costo de su establecimiento es demasiado alto para poder ser amortizado por las contribuciones indirectas de los usuarios, aunque esto no parece ser el caso del aeropuerto de El Altet, conforme podremos analizar más adelante.

El Altet, con sus 300 ha de terrenos ocupados — de los cuales unos $50.000 \mathrm{~m}^{2}$ corresponden a las dependencias de sus terminales de viajeros y mercancías- y los 2.678.091 pasajeros de 1991, entraría dentro de una categoría de aeropuerto a nivel internacional de tipo medio; es decir, está considerado como muy proporcionado en relación con su tráfico.

Su ubicación se realizó en su día siguiendo los criterios al uso en este tipo de instalaciones, proximidad a dos grandes urbes - Alicante y Elche - y en una zona que no presentaba concentración de población en sus inmediaciones (MORALES, 1990). Esta segunda motivación obliga, cuando se trata de grandes ciudades con área metropolitana anexa, a ubicaciones distantes de 50 a $70 \mathrm{~km}$ de los núcleos urbanos. No fue, ni es éste, el caso de El Altet que pudo emplazarse a sólo $10 \mathrm{~km}$ de Alicante y $15 \mathrm{~km}$ de Elche, con una zona de influencia, en su día, de unos $70 \mathrm{~km}$ de radio correspondiente a la isocrona de 60 minutos - que en la actualidad, con la notable mejora de la infraestructura viaria terrestre provincial y regional (autovía AlicanteMurcia, circunvalación de Alicante y autovía Alicante-Madrid y sus correspondientes enlaces con el aeropuerto y la A-7), pueden suponer un incremento de la zona de influencia, hasta un radio de unos $120 \mathrm{~km}$, que por el NE interfiere con el área de atracción de Manises, engloba todos los municipios industriales del Valle del Vinalopó y del Corredor de Almansa, y abarca por el W la Huerta de Murcia, Cartagena y el complejo La Manga-Mar Menor, constriñendo considerablemente el rendimiento y operatividad comercial del aeropuerto de la Comunidad Autónoma murciana (San Javier), peor comunicado con la ciudad y de menor importancia que El Altet.

Se trata de un área de influencia de alta densidad demográfica — casi 2,5 millones de habitantes- y con una economía floreciente centrada en actividades agrícolas, industriales, mercantiles y turísticas que la convierten en una de las más dinámicas a nivel nacional, ubicación que ha merecido los mejores calificativos en un reciente trabajo de investigación, —el Triángulo Alicante-Elche-Santa Pola—, realizado por la Universidad y patrocinado por el Club de Inversores.

Estos últimos datos indican que la importancia del aeropuerto alicantino deriva de su situación central respecto al ámbito turístico litoral de la Costa Blanca, ya que algo menos de una cuarta parte de su movimiento se justifica en relación con otras actividades socioeconómicas de su zona de atracción. Tiene que soportar una dura competencia del ferrocarril y del transporte por carretera, más rentable tanto desde un punto de vista de infraestructuras necesarias para su funcionamiento, como desde la variable tiempo, que era la que en parte se esgrimía para justificar su utilización en distancias medias. Por tanto, la 

solución pasa por acortar los tiempos de traslado a las terminales aéreas y ello a través de un adecuada red de autovías, como es el caso del aeropuerto de El Altet, muy mejorado en sus accesos por la nueva red viaria alicantina.

\section{Análisis del tráfico de pasajeros y mercancías}

En lo que hace referencia al tráfico de pasajeros y aeronaves, el aeropuerto de El Altet, desde su inauguración en 1967, ha participado en una serie de fluctuaciones que quedan reflejadas en los gráficos adjuntos, y que nos permiten comprobar, en relación a la última década, que:

- El tráfico nacional desde 1981 prácticamente se mantiene estacionado, tanto en aeronaves como en pasajeros, con una sensible mejora desde 1989.

- El tráfico internacional, desde 1985, ha operado un acusado incremento, tanto en aeronaves como pasajeros, con una caída preocupante en 1989 que parece superarse lentamente en los primeros meses de 1992.

- El incremento del número de naves en estos últimos años fue proporcionalmente menor que el número de pasajeros, lo cual refleja un mayor coeficiente de ocupación de las aeronaves, al tiempo que una mayor capacidad de las mismas (B-757, MD-80, por ejemplo). 
En este sentido, es necesario hacer mención a la íntima conexión entre el fenómeno charter y el turismo, muy patente en el aeropuerto alicantino. En efecto, el avión es el medio de transporte que cobra una importancia primordial dentro del turismo, tanto por la rapidez, como por la comodidad del desplazamiento. La mayoría del tráfico turístico se realiza en vuelos no regulares; vuelos «charter» que representan para el usuario una serie de ventajas:

- Suponen una reducción de tarifas muy sustancial, que los hace asequibles a un mayor número de viajeros.

- La mayoría son realizados por contratos con agencias de viajes, lo que implica que el viajero no sólo obtiene el servicio del desplazamiento, sino también estancia, hoteles, etc. Pero estos vuelos tienen su contrapartida; en muchos casos, no se realizan con aviones de «primer orden» sino aprovechando flotas marginales y con un elevado índice de ocupación, que se traduce en una mayor incomodidad en el desplazamiento.

Los vuelos no regulares representan una importante cifra en El Altet. De los 1.876.147 pasajeros de tráfico internacional en 1991, el 89,6\% corresponde a vuelo charter. Este tipo de tráfico no tiene importancia en lo que respecta a vuelos interiores donde el régimen charter se eleva sólo a 105.828 pasajeros, que representan sólo el 13,2\% del total de tráfico nacional de este aeropuerto.

Otro hecho que pone de relieve la importancia del tráfico turístico, dentro del movimiento general de pasajeros por vía aérea, es la fluctuación mensual del tráfico. En el 
período considerado (1988-1991), hemos podido observar un aumento del tráfico de aeronaves en los meses estivales y la consiguiente disminución en época invernal. En este sentido, el mes de febrero presenta el índice de tráfico más bajo tanto en vuelos regulares como en charter, tanto en interior como en exterior; por la misma razón, es obviamente el mes de menor desplazamiento de viajeros. Por el contrario, el semestre mayo-octubre destaca por sus altas concentraciones.

En cuanto a concurrencia empresarial, más de 60 Compañías Aéreas comerciales, en tráfico regular y charter, han venido operando en el aeropuerto de El Altet en los últimos años. El análisis de los datos correspondientes al último bienio (1990-91), así como al primer cuatrimestre de 1992, permite observar lo siguiente:

- El predominio lógico, por el carácter de monopolio, ejercido por IBERIA, en lo que se refiere a vuelos nacionales (768.946 pasajeros en 1990 y 543.433 en 1991); recesión explicable porque desde noviembre de 1991, esta compañía redujo su presencia en El Altet en favor de AVIACO que consiguientemente incrementó sus 

cifras de tráfico (30.735 pasajeros en el primer cuatrimestre de 1991, y 193.695 en el mismo período de 1992).

- La hegemonía en el tráfico internacional de la compañía BRITANNIA, que en 1990 movilizó en El Altet un total de 443.863 pasajeros, incrementados a 480.122 en el último año. En el primer cuatrimestre de 1992 se ha podido constatar un incremento del $16.4 \%$ con relación al mismo período del año anterior (148.477 entre enero y abril de 1991; y 172.858 en el presente año).

- El equilibrio de tráfico que muestran otras compañías dedicadas al vuelo charter como son DANAIR, SOBELAIR, MONARCH que mantienen sus cifras de tráfico con insensibles variaciones.

- La existencia de otras compañías que no ofrecen la regularidad de tráfico de las anteriores aunque movilizan cifras globales importantes, tal es el caso de AIR 2000, TRANSWEDE, TRANSAVIA, VIVA AIR, entre otras.

La importancia relativa que muestran las distintas compañías aéreas que operan en El Altet viene a ser reflejo de la distribución espacial del tráfico de pasajeros que, en el caso de vuelos internacionales, ofrece un total predominio de pasaje británico, mientras que el resto del pasaje europeo-internacional queda repartido, a notable distancia del británico, por holandeses (AIR HOLLAND), belgas (SOBELAIR), alemanes, escandinavos (TRANSWEDE), entre otros. 
Finalmente, indicar que el tráfico de mercancías en el aeropuerto de El Altet ha sufrido una acusada recesión en el período comprendido entre enero de 1990 y abril de 1992. En efecto, mientras que en el primero de los años considerados se cuantifican $6.671 .447 \mathrm{~kg}$ de mercancías, una estimación basada en el movimiento del primer cuatrimestre de 1992 alcanzaría como máximo los cuatro millones de kilogramos, lo cual supone una pérdida aproximada de casi el 40\%. En este sentido, los $4.901 .657 \mathrm{~kg}$ de 1991, ya suponen una merma del $-23,9 \%$ con referencia al año anterior.

La evolución y perspectiva futura que ofrece este tipo de tráfico en el aeropuerto de El Altet son las siguientes:

- En lo que concierne a la llegada, los fletes de mercancías entradas por el aeropuerto de Alicante en el bienio 1990-91 reflejan una disminución global de 210.317 kg, que suponen una regresión del 7,9\% con referencia al tráfico mercantil de 1990.

- En salidas, una acusada caída de los fletes: unos $1.283 .545 \mathrm{~kg}$, es decir, más del $-34 \%$ de regresión respecto a las cifras de 1990 en este mismo concepto. 
En conclusión, una bajada importante en el total global de fletes de 1991: se pierden casi un millón y medio de kilogramos en un sólo año. Tendencia regresiva en el valor en peso de los fletes de mercancías que parece corroborarse en el primer cuatrimestre de 1992, - signo evidente de que no es éste el objetivo de tráfico que parece definir en un futuro el aeropuerto de El Altet, mejor predispuesto evidentemente, a una funcionalidad relacionada con el sector turismo-.

\section{Consideraciones finales}

De todo lo analizado hasta ahora se pueden desprender las siguientes conclusiones:

1. Una tendencia al alza en función de los datos sobre tráfico de pasajeros en los cinco primeros meses de 1992, que reflejan una cierta recuperación de El Altet como centro receptor de pasajeros. En este sentido, se observa una cierta atenuación del carácter estacionario en el tráfico charter, al tiempo que se aprecia, asimismo, un movimiento de pasaje equilibrado entre vuelos nacionales e internacionales.

2. Una caída de los fletes mercantiles, con un decrecimiento bastante sensible del tráfico de mercancías; en este punto, la regresión ha alcanzado en 1991 la cota más baja de los últimos diez años.

3. El elevado porcentaje del tráfico internacional británico, en la modalidad charter fundamentalmente, sobre el total internacional, nos hace pensar en la necesidad de que por parte de los sectores turísticos se intente captar flujos procedentes de otros países, ya que con la actual «dependencia» del Reino Unido, cualquier variación en el comportamiento de este mercado, podría tener una profunda repercusión no sólo para el aeropuerto de El Altet, sino, obviamente, para el sector turístico de su zona de influencia (desde Denia a La Manga).

Por último, ante el peligro que, sobre el futuro del aeropuerto de El Altet, pueda suponer la aplicación de las directrices de la CE sobre reducción de puntos fronterizos y, consiguientemente, su desvalorización y pérdida de categoría internacional se propone la elaboración de un Plan Estratégico redactado por un equipo en el que aparezcan representados todos los estamentos administrativos, políticos y sociales de la provincia, que propugne un documento consensuado que, entre otros, establezca los siguientes puntos:

- Que las inversiones previstas para las instalaciones aeroportuarias de Alicante se ejecuten a la mayor brevedad posible, porque sólo si resulta competitivo podrá el aeropuerto de El Altet salvar las múltiples presiones que puedan generar su depreciación.

- Que se favorezca por parte de las empresas concurrentes en el tráfico aéreo del aeropuerto, la implantación de horarios de enlaces con las principales ciudades comerciales nacionales (Madrid, Sevilla, Bilbao, Barcelona,..) que permitan viajes de ida y vuelta en la misma jornada, si es menester utilizando aeronaves de menor capacidad que permitan abaratar los precios y hacer competitivos los costos.

- Que se estudie la posibilidad de enlazar la terminal del aeropuerto con la línea férrea Alicante-Murcia, que en la actualidad pasa a tan sólo 300 ó $400 \mathrm{~m}$ al norte del aparcamiento. Ese enlace, similar al existente en muchos aeropuertos europeos (Frankfurt, por ejemplo), posibilitaría un aprovechamiento más coherente de la infraestructura ferroviaria, al tiempo que mejoraría sensiblemente los sistemas de transferencia. 
- Que se tome en consideración el carácter de El Altet como AEROPUERTO SUPRARREGIONAL, apoyado por una infraestructura viaria terrestre que le permite abarcar un área de influencia, próxima —en algún caso- a los $120 \mathrm{~km}$, es decir, dentro de la isocrona de una hora; y en el centro de un área de considerable porvenir (como pone de manifiesto el reciente estudio sobre el Triángulo Alicante-ElcheSanta Pola), sobre el que puede verger todo el tráfico aéreo turístico con destino en la Costa Blanca.

\section{Bibliografía}

BELOTTI, J. (1975): L'economie du transport aérien, Belotti-Chatillon.

BODEGA FERNÁNDEZ, M ${ }^{a}$ D. (1979): «Veinte años en la evolución del tráfico aéreo en las diferentes áreas españolas», Actas del VI Congreso de Geografía, Palma de Mallorca, pp. 491493.

CANO GARCÍA, G. (1980): El transporte aéreo en España, ARIEL, Barcelona.

CÓRDOBA ORDÓÑEZ, J. (1976): «Evolución reciente de la red nacional de vuelos regulares de pasajeros en España (1970-75), Boletín de la Real Sociedad Geográfica, Madrid, pp. 169-184.

DACHARRY, M. (1981): Géographie du transport aérien, LITEC, París.

DASTIS QUECEDO, M. (1973), «El aeropuerto de Madrid-Barajas», Estudios Geográficos, vol. XXXIV, pp. 303-358.

GARCÍA ALCOLEA, R. y SERVET, J. (1983): «El transporte aéreo en el mundo y en España», Información Comercial Española, n. ${ }^{\circ}$ 594, pp. 27-36.

GIMÉNEZ Y CAPDEVILA, R. (1986): La geografía de los transportes en busca de su identidad, Geo-Crítica, Barcelona, 64 pp.

LABASSE, J.: La organización del espacio, IEAL, Madrid.

MORALES GIL, A. (1983): «La red viaria en el área metropolitana de Alicante-Elche», Investigaciones Geográficas, Inst. Universitario de Geografía. Alicante.

- (1985), «Accesibilidad por carretera al Aeropuerto de Alicante» Canelobre, Inst. «Juan GilAlbert», Diputación de Alicante.

- (Director; 1990, inéd.): Dinámica relacional Alicante-Elche. Impacto de nuevas comunicaciones. Dpto. de Análisis Geográfico Regional-Ayuntamiento de Alicante.

PEDREÑO, A. y FUSTER, B. (1993): «Perspectivas del aeropuerto del Altet en relación a la base económica de su área de influencia», Informe, $\mathrm{n}^{\circ}{ }^{\circ}$, Departamento de Análisis Económico Aplicado, Universidad de Alicante.

PIÑEIRO PELETEIRO, R. (1987): Comercio y transporte, Síntesis, Madrid.

SANZ GARCÍA, (1979): «Algunas reflexiones geográficas ante la reducción del espacio terrestre/ tiempo en virtud del transporte aéreo», Actas del VI Congreso de Geografía, Palma de Mallorca, pp. 605-611.

WOLKOWITSCH, M. (1973): Géographie des transports, Colin, París. 